\title{
ON THE SUMMABILITY OF THE DISCRETE HILBERT TRANSFORM
}

\author{
Rashid A. Aliev \\ Baku State University, Baku, AZ 1148, Azerbaijan; \\ Institute of Mathematics and Mechanics, NAS of Azerbaijan, \\ Baku, AZ 1141, Azerbaijan \\ aliyevrashid@mail.ru \\ Aynur F. Amrahova \\ Baku State University, Baku, AZ 1148, Azerbaijan \\ amrahovaaynur@mail.ru
}

\begin{abstract}
In this paper, we study the asymptotic behavior of the distribution function of the discrete Hilbert transform of sequences from the class $l_{1}$ and find a necessary condition and a sufficient condition for the summability of the discrete Hilbert transform of a sequence from the class $l_{1}$.

Keywords: Discrete Hilbert transform, Asymptotic behavior of the distribution function, Class of summable
\end{abstract} sequences.

\section{Introduction}

Denote by $l_{p}, p \geq 1$, the class of numeric sequences $b=\left\{b_{n}\right\}_{n \in Z}$ satisfying the condition

$$
\|b\|_{l_{p}}=\left(\sum_{n \in Z}\left|b_{n}\right|^{p}\right)^{1 / p}<\infty
$$

where $Z$ is the set of integers.

Let $b=\left\{b_{n}\right\}_{n \in Z} \in l_{1}$. The sequence $H(b)=\left\{(H b)_{n}\right\}_{n \in Z}$ is called the Hilbert transform of the sequence $b=\left\{b_{n}\right\}_{n \in Z}$, where

$$
(H b)_{n}=\sum_{m \neq n} \frac{b_{m}}{n-m}, \quad n \in Z .
$$

M. Riesz proved (see [10] and $[4,7]$ ) that, if $b \in l_{p}, p>1$, then $H(b) \in l_{p}$ and the inequality

$$
\|H(b)\|_{l_{p}} \leq C_{p}\|b\|_{l_{p}}
$$

holds. Weighted analogues of $(0.1)$ were investigated in $[1-3,5,6,8,9,11]$.

If $b \in l_{1}$, then the sequence $H(b)$ belongs to the class $\bigcap_{p>1} l_{p}$ but doesn't belong to the class $l_{1}$. In this case, R. Hunt, B. Muckenhoupt, and R. Wheeden proved (see [6]) that the distribution function

$$
(H b)(\lambda) \equiv \sum_{\left\{n \in Z:\left|(H b)_{n}\right|>\lambda\right\}} 1
$$


of the Hilbert transform of the sequence $b$ satisfies the condition

$$
\forall \lambda>0 \quad|(H b)(\lambda)| \leq \frac{C_{0}}{\lambda}\|b\|_{l_{1}},
$$

where $C_{0}$ is an absolute constant.

In this paper, we study the asymptotic behavior of the distribution function $(H b)(\lambda)$ of the Hilbert transform of a sequence $b \in l_{1}$ as $\lambda \rightarrow 0$ and find a necessary condition and a sufficient condition for the summability of the discrete Hilbert transform of a sequence from the class $l_{1}$.

\section{Asymptotic behavior of the distribution function of the discrete Hilbert transform}

Theorem 1. Let $b \in l_{1}$. Then the following equation holds:

$$
\lim _{\lambda \rightarrow 0+} \lambda \cdot(H b)(\lambda)=2\left|\sum_{n \in Z} b_{n}\right| .
$$

We first prove an auxiliary lemma.

Lemma 1. Let $b \in l_{1}$ and $\sum_{n \in Z} b_{n}=0$. Then the following equation holds:

$$
(H b)(\lambda)=o(1 / \lambda), \quad \lambda \rightarrow 0+.
$$

$\mathrm{P}$ r o o f. Assume first that the sequence $b \in l_{1}$ is concentrated on some finite interval $[-m, m]$, i. e., $b_{n}=0$ for $|n|>m$. In this case, from the equality

$$
(H b)_{n}=\sum_{|k| \leq m} \frac{b_{k}}{n-k}-\frac{1}{n-1 / 2} \sum_{|k| \leq m} b_{k}=\sum_{|k| \leq m} \frac{k-1 / 2}{(n-k)(n-1 / 2)} b_{k}, \quad|n|>m
$$

we get that

$$
\left|(H b)_{n}\right| \leq \frac{4}{n^{2}} \sum_{|k| \leq m}(k-1 / 2) b_{k}
$$

for large values of $n$, whence the asymptotic equation (1.2) follows.

Let us now consider the general case. From the condition $\sum_{n \in Z} b_{n}=0$, it follows that, for all $\varepsilon>0$ there exist sequences $b^{\prime}=\left\{b_{n}^{\prime}\right\}_{n \in Z} \in l_{1}$ and $b^{\prime \prime}=\left\{b_{n}^{\prime \prime}\right\}_{n \in Z} \in l_{1}$ satisfying the condition $b=b^{\prime}+b^{\prime \prime}$, where the sequence $b^{\prime} \in l_{1}$ is concentrated on some finite interval $[-m, m]$ and $\sum_{n \in Z} b_{n}^{\prime}=0$, and the sequence $b^{\prime \prime} \in l_{1}$ satisfies the inequality $\sum_{n \in Z}\left|b_{n}^{\prime \prime}\right|<\varepsilon /\left(4 C_{0}\right)$, with the constant $C_{0}$ from (0.2). Since the sequence $b^{\prime} \in l_{1}$ is concentrated on $[-m, m]$ and $\sum_{n \in Z} b_{n}^{\prime}=0$, equation (1.2) is satisfied for the sequence $b^{\prime} \in l_{1}$, and, therefore, there exists $\lambda(\varepsilon)>0$ such that the inequality

$$
\lambda\left(H b^{\prime}\right)(\lambda / 2)<\varepsilon / 2
$$

holds for $0<\lambda<\lambda(\varepsilon)$, where $\left(H b^{\prime}\right)(\lambda)=\sum_{\left\{n \in Z:\left|\left(H b^{\prime}\right)_{n}\right|>\lambda\right\}}$ 1. On the other hand, inequality (0.2) implies that

$$
\lambda\left(H b^{\prime \prime}\right)(\lambda / 2) \leq 2 C_{0} \sum_{n \in Z}\left|b_{n}^{\prime \prime}\right|<\varepsilon / 2
$$

for all $\lambda>0$, where $\left(H b^{\prime \prime}\right)(\lambda)=\sum_{\left\{n \in Z:\left|\left(H b^{\prime \prime}\right)_{n}\right|>\lambda\right\}}$ 1. From inequalities (1.3) and (1.4) and the inclusion

$$
\left\{n \in Z:\left|(H b)_{n}\right|>\lambda\right\} \subset\left\{n \in Z:\left|\left(H b^{\prime}\right)_{n}\right|>\lambda / 2\right\} \bigcup\left\{n \in Z:\left|\left(H b^{\prime \prime}\right)_{n}\right|>\lambda / 2\right\}
$$


we obtain that

$$
\lambda \cdot(H b)(\lambda) \leq \lambda\left(H b^{\prime}\right)(\lambda / 2)+\lambda\left(H b^{\prime \prime}\right)(\lambda / 2)<\varepsilon
$$

for $0<\lambda<\lambda(\varepsilon)$. This shows that equality (1.2) holds for all $b \in l_{1}$ satisfying the condition $\sum_{n \in Z} b_{n}=0$. This completes the proof of Lemma 1 .

P r o o f of Theorem 1. In the case $\sum_{n \in Z} b_{n}=0$, the statement of the theorem follows from Lemma 1. Consider the case $\sum_{n \in Z} b_{n}=\alpha \neq 0$. We use the following notation: $b_{n}^{\prime}=b_{n}$ for $n \neq 0$, $b_{0}^{\prime}=b_{0}-\alpha, b_{n}^{\prime \prime}=0$ for $n \neq 0$, and $b_{0}^{\prime \prime}=\alpha$. Then $b=b^{\prime}+b^{\prime \prime}$, where $b^{\prime}=\left\{b_{n}^{\prime}\right\}_{n \in Z} \in l_{1}$ and $b^{\prime \prime}=\left\{b_{n}^{\prime \prime}\right\}_{n \in Z} \in l_{1}$. Since $\sum_{n \in Z} b_{n}^{\prime}=0$, we obtain from Lemma 1 that

$$
\left(H b^{\prime}\right)(\lambda)=o(1 / \lambda), \quad \lambda \rightarrow 0+.
$$

Since $\left(H b^{\prime \prime} t\right)_{n}=\alpha / n$ for $n \neq 0$ and $\left(H b^{\prime \prime}\right)_{0}=0$, we have

$$
\left(H b^{\prime \prime}\right)(\lambda) \sim \frac{2|\alpha|}{\lambda}, \quad \lambda \rightarrow 0+.
$$

For all $0<\varepsilon<1$, by the inclusions

$$
\begin{aligned}
& \left\{n \in Z:\left|\left(H b^{\prime \prime}\right)_{n}\right|>(1+\varepsilon) \lambda\right\} \backslash\left\{n \in Z:\left|\left(H b^{\prime}\right)_{n}\right|>\varepsilon \lambda\right\} \subset \\
& \subset\left\{n \in Z:\left|(H b)_{n}\right|>\lambda\right\} \subset \\
& \subset\left\{n \in Z:\left|\left(H b^{\prime}\right)_{n}\right|>\varepsilon \lambda\right\} \bigcup\left\{n \in Z:\left|\left(H b^{\prime \prime}\right)_{n}\right|>(1-\varepsilon) \lambda\right\}
\end{aligned}
$$

and relations (1.5) and (1.6), we have

$$
\frac{2|\alpha|}{1+\varepsilon} \leq \liminf _{\lambda \rightarrow 0+} \lambda \cdot(H b)(\lambda) \leq \limsup _{\lambda \rightarrow 0+} \lambda \cdot(H b)(\lambda) \leq \frac{2|\alpha|}{1-\varepsilon}
$$

This implies equation (1.1) and completes the proof of Theorem 1.

\section{A necessary condition and a sufficient condition for the summability of the discrete Hilbert transform}

Theorem 2. Let $b \in l_{1}$. If $H b \in l_{1}$, then it is necessary that the following equation holds:

$$
\sum_{n \in Z} b_{n}=0
$$

P r o o f. We first we prove that, if $h=\left\{h_{n}\right\}_{n \in Z} \in l_{1}$, then the distribution function $h(\lambda)=\sum_{\left\{n \in Z:\left|h_{n}\right|>\lambda\right\}} 1$ of the sequence $h$ satisfies the condition

$$
h(\lambda)=o(1 / \lambda), \quad \lambda \rightarrow 0+.
$$

Note that the condition $h=\left\{h_{n}\right\}_{n \in Z} \in l_{1}$ implies that the set of $\left\{n \in Z:\left|h_{n}\right|>\lambda\right\}$ is finite for all $\lambda>0$. Then, the inequality

$$
\sum_{n \in Z}\left|h_{n}\right|=\sum_{\left\{n \in Z:\left|h_{n}\right|>1\right\}}\left|h_{n}\right|+\sum_{k=0}^{\infty}\left[\sum_{\left\{n \in Z:\left|h_{n}\right| \in\left(2^{-k-1} ; 2^{-k}\right]\right\}}\left|h_{n}\right|\right] \geq
$$




$$
\begin{gathered}
\geq \sum_{\left\{n \in Z:\left|h_{n}\right|>1\right\}} 1+\sum_{k=0}^{\infty}\left[\sum_{\left\{n \in Z:\left|h_{n}\right| \in\left(2^{-k-1} ; 2^{-k}\right]\right\}} 2^{-k-1}\right]= \\
=h(1)+\sum_{k=0}^{\infty}\left[2^{-k-1} \cdot\left(h\left(2^{-k-1}\right)-h\left(2^{-k}\right)\right)\right]=\sum_{k=0}^{\infty}\left[2^{-k-1} \cdot h\left(2^{-k}\right)\right]
\end{gathered}
$$

implies that

$$
\lim _{k \rightarrow \infty} 2^{-k} \cdot h\left(2^{-k}\right)=0
$$

Hence, taking into account that the function $h(\lambda)$ is decreasing, we obtain (2.2).

It follows from (2.1) that, if $H b \in l_{1}$, then

$$
(H b)(\lambda)=o(1 / \lambda), \quad \lambda \rightarrow 0+,
$$

and, therefore, by Theorem 1, we obtain that the equation (2.2) holds. The proof of Theorem 2 is complete.

Theorem 3. If asequence $b \in l_{1}$ satisfies the conditions

(i) $\sum_{n \in Z} b_{n}=0$

(ii) $\sum_{m \in Z}^{n \in Z}\left|b_{m}\right| \ln (e+|m|)<\infty$, then $H b \in l_{1}$ and the following inequality holds:

$$
\|H b\|_{l_{1}} \leq 6 \sum_{m \in Z}\left|b_{m}\right| \ln (e+|m|) .
$$

P r o o f. It follows from the definition of the discrete Hilbert transform that

$$
\left|(H b)_{0}\right|=\left|\sum_{m \neq 0} \frac{b_{m}}{m}\right| \leq\|b\|_{l_{1}} .
$$

From condition $(i)$ for $n \neq 0$, we obtain that

$$
\left|(H b)_{n}\right|=\left|\sum_{m \neq n} \frac{b_{m}}{n-m}\right|=\left|\sum_{m \neq n} \frac{b_{m}}{n-m}-\sum_{m \neq n} \frac{b_{m}}{n}-\frac{b_{n}}{n}\right| \leq\left|\frac{b_{n}}{n}\right|+\sum_{m \neq n} \frac{|m|\left|b_{m}\right|}{|n||n-m|} .
$$

It follows from inequalities (2.4) and (2.5) that

$$
\begin{gathered}
\|H b\|_{l_{1}}=\sum_{n \in Z}\left|(H b)_{n}\right| \leq 2\|b\|_{l_{1}}+\sum_{n \neq 0}\left[\sum_{m \neq n} \frac{|m|\left|b_{m}\right|}{|n||n-m|}\right]= \\
=2\|b\|_{l_{1}}+\sum_{n>0}\left[\sum_{m>n} \frac{|m|\left|b_{m}\right|}{|n||n-m|}\right]+\sum_{n>0}\left[\sum_{m<n} \frac{|m|\left|b_{m}\right|}{|n||n-m|}\right]+ \\
+\sum_{n<0}\left[\sum_{m>n} \frac{|m|\left|b_{m}\right|}{|n||n-m|}\right]+\sum_{n<0}\left[\sum_{m<n} \frac{|m|\left|b_{m}\right|}{|n||n-m|}\right]= \\
=2\|b\|_{l_{1}}+J_{1}+J_{2}+J_{3}+J_{4} .
\end{gathered}
$$

Let us estimate the summands $J_{k}, k=1,2,3,4$. From condition $(i i)$ and f equalities of the form

$$
\sum_{n<0}\left(\frac{1}{n-m}-\frac{1}{n}\right)=\left(\frac{1}{-1-m}+1\right)+\left(\frac{1}{-2-m}+\frac{1}{2}\right)+\ldots+\left(\frac{1}{-m-m}+\frac{1}{m}\right)+
$$




$$
+\left(\frac{1}{-m-1-m}+\frac{1}{m+1}\right)+\left(\frac{1}{-m-2-m}+\frac{1}{m+2}\right)+\ldots=1+\frac{1}{2}+\ldots+\frac{1}{m},
$$

for $m>0$, and

$$
\begin{aligned}
& \sum_{n>0}\left(\frac{1}{n}-\frac{1}{n-m}\right)=\left(1-\frac{1}{1+|m|}\right)+\left(\frac{1}{2}-\frac{1}{2+|m|}\right)+\ldots+\left(\frac{1}{|m|}-\frac{1}{|m|+|m|}\right)+ \\
& +\left(\frac{1}{|m|+1}-\frac{1}{|m|+1+|m|}\right)+\left(\frac{1}{|m|+2}-\frac{1}{|m|+2+|m|}\right)+\ldots=1+\frac{1}{2}+\ldots+\frac{1}{|m|},
\end{aligned}
$$

for $m<0$, we obtain that

$$
\begin{aligned}
& J_{1}=\sum_{n>0}\left[\sum_{m>n} \frac{|m|\left|b_{m}\right|}{|n||n-m|}\right]=\sum_{m>1}\left[\sum_{0<n<m} \frac{m\left|b_{m}\right|}{n(m-n)}\right]= \\
& =\sum_{m>1}\left|b_{m}\right| \cdot\left[\sum_{0<n<m}\left(\frac{1}{m-n}+\frac{1}{n}\right)\right]=2 \sum_{m>1}\left|b_{m}\right| \cdot\left[1+\frac{1}{2}+\ldots+\frac{1}{m-1}\right] \leq \sum_{m>1}\left|b_{m}\right| \cdot \ln m, \\
& J_{2}=\sum_{n<0}\left[\sum_{m>n} \frac{|m|\left|b_{m}\right|}{|n||n-m|}\right]=\sum_{m>0}\left[\sum_{n<0} \frac{m\left|b_{m}\right|}{n(n-m)}\right]+\sum_{m<0}\left[\sum_{n<m} \frac{m\left|b_{m}\right|}{n(m-n)}\right]= \\
& =\sum_{m>0}\left|b_{m}\right| \cdot\left[\sum_{n<0}\left(\frac{1}{n-m}-\frac{1}{n}\right)\right]+\sum_{m<0}\left|b_{m}\right| \cdot\left[\sum_{n<m}\left(\frac{1}{m-n}+\frac{1}{n}\right)\right]= \\
& =\sum_{m>0}\left|b_{m}\right| \cdot\left[1+\frac{1}{2}+\ldots+\frac{1}{m}\right]+\sum_{m<0}\left|b_{m}\right| \cdot\left[1+\frac{1}{2}+\ldots+\frac{1}{|m|}\right] \leq \sum_{m \in Z}\left|b_{m}\right| \cdot \ln (1+|m|), \\
& J_{3}=\sum_{n>0}\left[\sum_{m<n} \frac{|m|\left|b_{m}\right|}{|n||n-m|}\right]=\sum_{m<0}\left[\sum_{n>0} \frac{m\left|b_{m}\right|}{n(m-n)}\right]+\sum_{m>0}\left[\sum_{n>m} \frac{m\left|b_{m}\right|}{n(n-m)}\right]= \\
& =\sum_{m<0}\left|b_{m}\right| \cdot\left[\sum_{n>0}\left(\frac{1}{n}-\frac{1}{n-m}\right)\right]+\sum_{m>0}\left|b_{m}\right| \cdot\left[\sum_{n>m}\left(\frac{1}{n-m}-\frac{1}{n}\right)\right]= \\
& =\sum_{m<0}\left|b_{m}\right| \cdot\left[1+\frac{1}{2}+\ldots+\frac{1}{|m|}\right]+\sum_{m>0}\left|b_{m}\right| \cdot\left[1+\frac{1}{2}+\ldots+\frac{1}{m}\right] \leq \sum_{m \in Z}\left|b_{m}\right| \cdot \ln (1+|m|), \\
& J_{4}=\sum_{n<0}\left[\sum_{m<n} \frac{|m|\left|b_{m}\right|}{|n||n-m|}\right]=\sum_{m<-1}\left[\sum_{m<n<0} \frac{m\left|b_{m}\right|}{n(n-m)}\right]= \\
& =\sum_{m<-1}\left|b_{m}\right| \cdot\left[\sum_{m<n<0}\left(\frac{1}{n-m}-\frac{1}{n}\right)\right]= \\
& =2 \sum_{m<-1}\left|b_{m}\right| \cdot\left[1+\frac{1}{2}+\ldots+\frac{1}{|m|-1}\right] \leq 2 \sum_{m<-1}\left|b_{m}\right| \cdot \ln |m| .
\end{aligned}
$$

From this and (2.6), we obtain (2.3). The proof of Theorem 3 is complete.

Theorem 4. The following equation holds under the conditions of Theorem 3:

$$
\sum_{n \in Z}(H b)_{n}=0
$$


P r o o f. By the conditions of Theorem 3,

$$
(H b)_{0}=-\sum_{m \neq 0} \frac{b_{m}}{m}
$$

and

$$
(H b)_{n}=\sum_{m \neq n} \frac{b_{m}}{n-m}=\sum_{m \neq n} \frac{b_{m}}{n-m}-\sum_{m \neq n} \frac{b_{m}}{n}-\frac{b_{n}}{n}=\sum_{m \neq n} \frac{m b_{m}}{n(n-m)}-\frac{b_{n}}{n}
$$

for $n \neq 0$. Therefore, we have

$$
\begin{gathered}
\sum_{n \in Z}(H b)_{n}=-\sum_{m \neq 0} \frac{b_{m}}{m}+\sum_{n \neq 0}\left[\sum_{m \neq n} \frac{m b_{m}}{n(n-m)}-\frac{b_{n}}{n}\right]=-2 \sum_{m \neq 0} \frac{b_{m}}{m}+\sum_{n \neq 0}\left[\sum_{m \neq n} \frac{m b_{m}}{n(n-m)}\right]= \\
=-2 \sum_{m \neq 0} \frac{b_{m}}{m}+\sum_{n>0}\left[\sum_{m>n} \frac{m b_{m}}{n(n-m)}\right]+\sum_{n>0}\left[\sum_{m<n} \frac{m b_{m}}{n(n-m)}\right]+ \\
+\sum_{n<0}\left[\sum_{m>n} \frac{m b_{m}}{n(n-m)}\right]+\sum_{n<0}\left[\sum_{m<n} \frac{m b_{m}}{n(n-m)}\right]=-2 \sum_{m \neq 0} \frac{b_{m}}{m}+j_{1}+j_{2}+j_{3}+j_{4} .
\end{gathered}
$$

It follows from condition $(i i)$ that

$$
\begin{gathered}
j_{1}=\sum_{n>0}\left[\sum_{m>n} \frac{m b_{m}}{n(n-m)}\right]=\sum_{m>1}\left[\sum_{0<n<m} \frac{m b_{m}}{n(n-m)}\right]= \\
=\sum_{m>1} b_{m} \cdot\left[\sum_{0<n<m}\left(\frac{1}{n-m}-\frac{1}{n}\right)\right]=-2 \sum_{m>1} b_{m} \cdot\left[1+\frac{1}{2}+\ldots+\frac{1}{m-1}\right], \\
j_{2}=\sum_{n<0}\left[\sum_{m>n} \frac{m b_{m}}{n(n-m)}\right]=\sum_{m>0}\left[\sum_{n<0} \frac{m b_{m}}{n(n-m)}\right]+\sum_{m<0}\left[\sum_{n<m} \frac{m b_{m}}{n(n-m)}\right]= \\
=\sum_{m>0} b_{m} \cdot\left[\sum_{n<0}\left(\frac{1}{n-m}-\frac{1}{n}\right)\right]+\sum_{m<0} b_{m} \cdot\left[\sum_{n<m}\left(\frac{1}{n-m}-\frac{1}{n}\right)\right]= \\
=\sum_{m>0} b_{m} \cdot\left[1+\frac{1}{2}+\ldots+\frac{1}{m}\right]-\sum_{m<0} b_{m} \cdot\left[1+\frac{1}{2}+\ldots+\frac{1}{|m|}\right], \\
j_{3}=\sum_{n>0}\left[\sum_{m<n} \frac{m b_{m}}{n(n-m)}\right]=\sum_{m<0}\left[\sum_{n>0} \frac{m b_{m}}{n(n-m)}\right]+\sum_{m>0}\left[\sum_{n>m} \frac{m b_{m}}{n(n-m)}\right]= \\
=\sum_{m<0} b_{m} \cdot\left[\sum_{n>0}\left(\frac{1}{n-m}-\frac{1}{n}\right)\right]+\sum_{m>0} b_{m} \cdot\left[\sum_{n>m}\left(\frac{1}{n-m}-\frac{1}{n}\right)\right]= \\
=-\sum_{m<0} b_{m} \cdot\left[1+\frac{1}{2}+\ldots+\frac{1}{|m|}\right]+\sum_{m>0} b_{m} \cdot\left[1+\frac{1}{2}+\ldots+\frac{1}{m}\right], \\
j_{4}=\sum_{n<0}\left[\sum_{m<n} \frac{m b_{m}}{n(n-m)}\right]=\sum_{m<-1}\left[\sum_{m<n<0} \frac{m b_{m}}{n(n-m)}\right]= \\
\sum_{m<-1} b_{m} \cdot\left[\sum_{m<n<0}\left(\frac{1}{n-m}-\frac{1}{n}\right)\right]=2 \sum_{m<-1} b_{m} \cdot\left[1+\frac{1}{2}+\ldots+\frac{1}{|m|-1}\right] .
\end{gathered}
$$

From this and (2.8), we obtain (2.7). The proof of Theorem 4 is complete. 


\section{Acknowledgements}

The authors thank the anonymous referees for careful reading of the manuscript and very useful comments. This research was supported by the Science Development Foundation under the President of the Republic of Azerbaijan (grant no. EIF/MQM/Elm-Tehsil-1-2016-1(26)-71/08/01).

\section{REFERENCES}

1. Andersen K.F. Inequalities with weights for discrete Hilbert transforms. Canad. Math. Bul., 1977. Vol. 20. P. 9-16.

2. Belov Y., Mengestie T. Y., Seip K. Discrete Hilbert transforms on sparse sequences. Proc. London Math. Soc., 2011. Vol. 103, No. 1. P. 73-105. DOI: 10.1112/plms/pdq053

3. Belov Y., Mengestie T. Y., Seip K. Unitary discrete Hilbert transforms. J. Anal. Math., 2010. Vol. 112. P. 383-393. DOI: 10.1007/s11854-010-0035-y

4. De Carli L., Samad S. One-parameter groups and discrete Hilbert transform. Canad. Math. Bull., 2016. Vol. 59. P. 497-507. arXiv: 1506.03362 [math.FA]. URL: https://arxiv.org/pdf/1506.03362.pdf

5. Gabisonija I., Meskhi A. Two weighted inequalities for a discrete Hilbert transform. Proc. A. Razmadze Math. Inst., 1998. Vol. 116. P. 107-122. URL: http://rmi.tsu.ge/proceedings/volumes/ps/v116-4.ps.gz

6. Hunt R., Muckenhoupt B., Wheeden R. Weighted norm inequalities for the conjugate function and Hilbert transform. Trans. Amer. Math. Soc., 1973. Vol. 176, No. 2. P. 227-251. DOI: 10.2307/1996205

7. Laeng E. Remarks on the Hilbert transform and some families of multiplier operators related to it. Collect. Math., 2007. Vol. 58, No. 1. P. 25-44.

URL: https://www.raco.cat/index.php/CollectaneaMathematica/article/view/57795

8. Liflyand E. Weighted Estimates for the Discrete Hilbert Transform. In: Methods of Fourier Analysis and Approximation Theory. Applied and Numerical Harmonic Analysis, ed. M. Ruzhansky, S. Tikhonov. Cham: Birkhäuser, 2016. P. 59-69. DOI: 10.1007/978-3-319-27466-9_5

9. Rakotondratsimba Y. Two weight inequality for the discrete Hilbert transform. Soochow J. Math., 1999. Vol. 25, No. 4. P. 353-373. URL: http://mathlab.math.scu.edu.tw/mp/pdf/S25N44.pdf

10. Riesz M. Sur les fonctions conjuguees. Math. Z., 1928. Vol. 27. P. 218-244.

URL: https://eudml.org/doc/167977

11. Stepanov V. D., Tikhonov S. Yu. Two weight inequalities for the Hilbert transform of monotone functions. Dokl. Math., 2011. Vol. 83, No. 2. P. 241-242. DOI: 10.1134/S1064562411020359 